\title{
Flow transitions in a rotating magnetic field
}

\author{
M. P. Volz, K. Mazuruk
}

\begin{tabular}{|c|c|}
\hline \multicolumn{2}{|c|}{$\begin{array}{l}\text { Abstract Critical Rayleigh numbers have been measured in } \\
\text { a liquid metal cylinder of finite height in the presence of a } \\
\text { rotating magnetic field. Several different stability regimes were } \\
\text { observed, which were determined by the values of the Rayleigh } \\
\text { and Hartmann numbers. For weak rotating magnetic fields and } \\
\text { small Rayleigh numbers, the experimental observations can } \\
\text { be explained by the existence of a single non-axisymmetric } \\
\text { meridional roll rotating around the cylinder, driven by the } \\
\text { azimuthal component of the magnetic field. The measured } \\
\text { dependence of rotational velocity on magnetic field strength is } \\
\text { consistent with the existence of laminar flow in this regime. }\end{array}$} \\
\hline \multicolumn{2}{|c|}{ List of symbols } \\
\hline$B_{0}$ & magnitude of magnetic \\
\hline$B_{r}, B_{\theta}$ & $\begin{array}{l}\text { radial and azimuthal magnetic induction com- } \\
\text { ponents }\end{array}$ \\
\hline C & wall admittance \\
\hline$d$ & cell diameter \\
\hline$d_{w}$ & wall thickness \\
\hline & gravity at earth's surface \\
\hline $\mathrm{Ha}$ & Hartmann number \\
\hline$h$ & cell height \\
\hline & thermal conductivity of fluid \\
\hline & thermal conductivity of wall \\
\hline$L 1, L 2, L 3, L 4$ & thermistor temperatures \\
\hline$R a$ & Rayleigh number \\
\hline$R a^{r}$ & $\begin{array}{l}\text { critical Rayleigh number for the transition from } \\
\text { no flow to laminar flow }\end{array}$ \\
\hline$R a^{t}$ & $\begin{array}{l}\text { critical Rayleigh number for the transition from } \\
\text { time-independent to time-dependent flow }\end{array}$ \\
\hline & radial coordinate \\
\hline & temperature at top of cell \\
\hline & temperature at bottom of cell \\
\hline
\end{tabular}

Received: 29 June 1995/Accepted: 18 October 1995

M. P. Volz, K. Mazuruk

Space Science Laboratory, NASA Marshall Space Flight Center, Huntsville, AL 35812, USA

Correspondence to: M. P.Volz

This work was supported by the Microgravity Science and Applications Division of the National Aeronautics and Space Administration.

\author{
$\Delta T \quad$ temperature difference between cell bottom \\ and cell top \\ $\Delta T_{c} \quad$ critical temperature difference between cell \\ bottom and top time \\ time \\ $U 1, U 2, U 3$, \\ U4 \\ $z$ \\ $\alpha$ \\ $\delta$

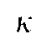 \\ $\mu$ \\ $v$ \\ $\rho$ \\ $\sigma$ \\ 0 \\ (1) \\ thermistor temperatures \\ vertical coordinate \\ volumetric thermal expansion coefficient \\ skin depth \\ thermal diffusivity \\ magnetic permeability \\ kinematic viscosity \\ density \\ electrical conductivity \\ azimuthal coordinate \\ angular frequency of magnetic induction
}

1

\section{Introduction}

There has been considerable interest lately in the applicatis, $n$ of rotating magnetic fields to the processes of single crystal growth (see, for example, Dold et al. 1995; Brückner and Schwerdtfeger 1994; Barmin et al. 1992). This has been motivated by the potential benefits that rotating magnetic fields can have on the crystallization process. These include stirring of multicomponent melts, enhancement of the interface stability, and a reduction of thermal asymmetry at the solidification front. In order to take advantage of these benefits, it is essential to understand the fluid flow of liquid metal under the influence of a rotating magnetic field. Indeed, there have been a number of papers devoted to this endeavor (Gelfgat et al. 1993; Davidson and Hunt 1987, Doronin et al. 1973). This paper presents experimental results of fluid flow behavior under the combined forces generated by rotating magnetic fields and gravity. To achieve this, a cylindrical test cell has been designed to make carefully controlled measurements of the flow transitions between the regimes that exist under these combined forces. These flow transitions are observed by measuring the temperature changes of thermistors immersed in the liquid metal. Critical Rayleigh numbers are measured for the case of $B_{0}=0$ for comparison to previous theoretical, numerical, and experimental results. Then, the critical Rayleigh numbers are measured in the presence of a rotating magnetic field, and the fluid behavior in the flow regimes identified are examined. 

2

\section{Experimental set-up}

The experiments were conducted using liquid gallium contained in a vertical cylindrical test cell depicted in Fig. 1. The side walls were made from polymethyl methacrylate (PMMA) and copper discs were used for the top and bottom. The temperatures of the top and bottom of the cell were controlled by fluid recirculating from constant temperature water baths. The transmission of vibrations between the fluid pumps and the test cell were reduced by first flowing the fluid through passive pressurized containers placed between the fluid pumps and the test cell. The temperatures of the cell top $\left(T_{a}\right)$ and cell bottom $\left(T_{b}\right)$ were measured by thermocouples placed in the copper disks less than $0.5 \mathrm{~mm}$ from the copper gallium interface. The cell height was $24 \mathrm{~mm}$, and the cell diameter was $23 \mathrm{~mm}$ for a resultant aspect ratio $h / d$ of 1.04 . Eight thermistors were placed in the cell to record temperature changes and infer from them the nature of the fluid flow. Four of the thermistors, labelled $L 1$ through $L 4$, were placed in a horizontal plane at $z=h / 3$, and the other four, labelled $U 1$ through $U 4$, were placed in a horizontal plane located at $z=2 h / 3$. The thermistors were positioned at azimuthal positions of $\theta=0, \pi / 2, \pi$, and $3 \pi / 2$. The diameters of the thermistors were $0.6 \mathrm{~mm}$ and they protruded into the melt a distance of $2 \mathrm{~mm}$. The thermistors were operated with a constant current of $10 \mu \mathrm{A}$ which generated negligible self-heating in the system. Both the thermocouples and thermistors were calibrated by recording their voltages under isothermal conditions at several temperatures spanning
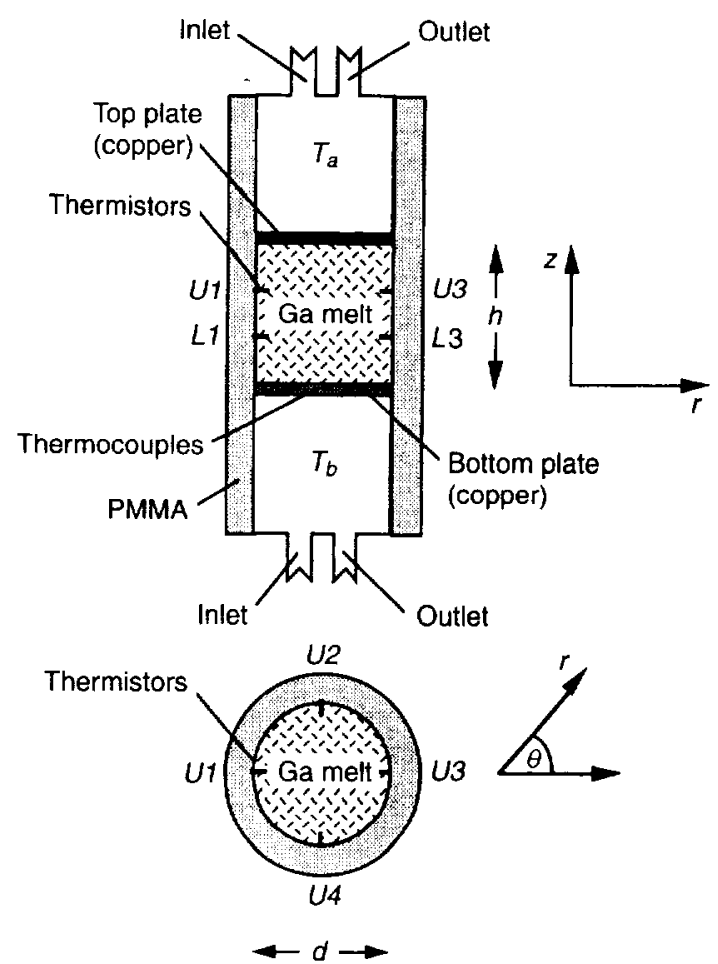

Fig. 1. Schematic drawing of the test cell. Shown at the top is a vertical cross section of the cell in the $r, z$ plane for $\theta=0$. The gravitational vector is in the $-z$ direction. At the bottom is a horizontal cross section of the cell in the $r /$ plane for $z=2 h / 3 . L 1, L 2, L 3, L 4$ and $U 1, U 2$, $U 3, U 4$ designate the thermistors immersed in the liquid gallium
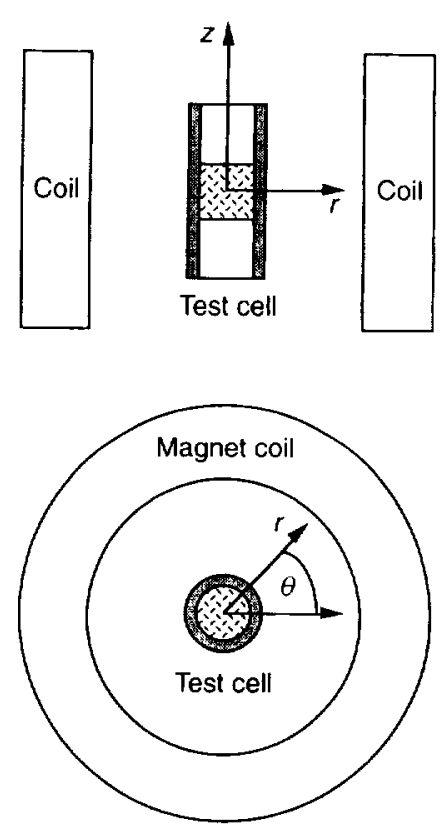

Fig. 2. A side and top view of the position of the test cell within the rotating magnetic field coil

the temperature range of interest. The thermocouple and thermistor signals were digitized with a 16-Bit analog-to-digital convertor (ADC) located in a slot of an AT-type PC and then recorded on computer disk. Up to 8 thermistor or thermocouple signals could be recorded simultaneously. The noise of the system was such that it was possible to obtain a temperature resolution of better than $2 \mathrm{mK}$ with the thermistors immersed in liquid gallium.

The test cell was placed inside a rotating magnetic field as sketched in Fig. 2. The field was generated by a three pole, three phase inductor operating at $60 \mathrm{~Hz}$. The magnet had a $120 \mathrm{~mm}$ inner diameter, a $180 \mathrm{~mm}$ outer diameter, and was $120 \mathrm{~mm}$ in height. The magnetic field profiles were measured with a Hall probe without the test cell present and the variation in field strength over both the vertical and horizontal dimensions of the cell was less than $3 \%$. The magnetic field rotated in the $r, \theta$ plane and can be described by the equations:

$B_{r}(t)=B_{0} \cos (\theta-\omega t), \quad B_{\theta}(t)=B_{0} \sin (\theta-\omega t)$

where $B_{0}$ is the magnitude of the applied magnetic field and $\omega$ is its angular frequency.

\section{3}

\section{Measurement of critical Rayleigh numbers}

The test cell was designed to accurately measure critical values of the Rayleigh number. The Rayleigh number is defined as

$R a=\frac{g \alpha\left(T_{b}-T_{a}\right) h^{3}}{\kappa \nu}$

where $g$ is the gravitational acceleration on the earth's surface, $9.81 \mathrm{~m} / \mathrm{s}^{2}, \alpha$ the volumetric thermal expansion coefficient, $T_{b}-T_{a}$ the vertical temperature difference across the cell, $h$ the cell height, $k$ the thermal diffusivity and $v$ the kinematic viscosity. Table 1 lists the material properties of liquid gallium 
Table 1.

\begin{tabular}{|c|c|c|c|c|}
\hline Property (323 K) & Symbol & Value & Units & Reference(s) \\
\hline Thermal expansion & $\alpha$ & $1.25 \times 10^{-4}$ & $1 / \mathrm{K}$ & Hoather (1936) \\
\hline Thermal diffusivity & $k$ & $1.38 \times 10^{-5}$ & $\mathrm{~m}^{2} / \mathrm{s}$ & $\begin{array}{l}\text { Iida and Guthrie (1988) } \\
\text { Powell and Childs (1974) }\end{array}$ \\
\hline Kinematic viscosity & $v$ & $3.177 \times 10^{-7}$ & $\mathrm{~m}^{2} / \mathrm{s}$ & Grosse (1961) \\
\hline Density & $\rho$ & $6.075 \times 10^{3}$ & $\mathrm{~kg} / \mathrm{m}^{3}$ & $\begin{array}{l}\text { Nalgiev and Ibragimov } \\
\text { (1974) }\end{array}$ \\
\hline Electrical conductivity & $\sigma$ & $3.82 \times 10^{6}$ & $\mathrm{~S} / \mathrm{m}$ & Cusack and Kendall (1960) \\
\hline
\end{tabular}

at $323 \mathrm{~K}$ necessary to calculate the Rayleigh number and the interaction of liquid gallium with a rotating magnetic field. The thermal diffusivity was calculated from the thermal conductivity given by Powell and Childs (1972) and the heat capacity found in Iida and Guthrie (1988). The material properties $\alpha, k$, and $v$ vary as a function of temperature. However, the quantity $\alpha / k v$ varies by less than $3 \%$ over the range of temperatures at which the critical Rayleigh numbers were measured. Therefore, all calculations of the Rayleigh number assumed that the material properties were temperature-independent and utilized the values listed in Table 1.

The critical Rayleigh number $R a^{r}$ is generally defined to be the transition value between the static condition state and the time-independent laminar convection state. The critical Rayleigh number $R a^{\prime}$ is the value where the transition between time-independent steady convection and time-dependent convection occurs. These critical values were measured here to compare to previous theoretical results and as a baseline for comparison of these transitions in the presence of a rotating magnetic field.

Two methods were used to measure the critical Rayleigh numbers. One method consisted of starting with a vertical temperature difference $\Delta T$ less than the critical $\Delta T$, keeping $T_{a}$ constant, and slowly raising the temperature of the cylinder bottom $T_{b}$. The second method consisted of starting with a $\Delta T$ larger than the critical $\Delta T$, keeping $T_{a}$ constant, and slowly lowering $T_{b}$. As pointed out by Müller and Neumann (1983), it is necessary to keep the heating rate quasistatic, so that the temperature profile in the fluid remains linear. This condition can be met as long as

$\frac{\mathrm{d} T}{\mathrm{~d} t}<\frac{2 A T_{c} \kappa}{h^{2}}$

where $\mathrm{d} T / \mathrm{d} t$ is the heating rate and $A T_{c}$ is the temperature difference $T_{b}-T_{a}$ when the critical Rayleigh number is reached. For a low Prandtl number fluid such as gallium, this criterion is easily met, and for the present experimental set-up, this criterion dictates that $\mathrm{d} T / \mathrm{d} t<5 \times 10^{-2} \mathrm{~K} / \mathrm{s}$.

The experimental results suggest that Eq. (3) is a necessary but not sufficient condition to obtain an accurate measurement of $R a^{c}$. There is an additional condition which occurs as a result of fluid inertia. Consider a fluid at rest and placed in the temperature bath, so that $R a>R a^{c}$. Then the rest state is unstable, and it will relax to the convection state. The time constant of this transition is approximately proportional to the difference $R a-R a$. Retardation effects for the fluid flow rearrangement will be seen for any finite temperature ramping

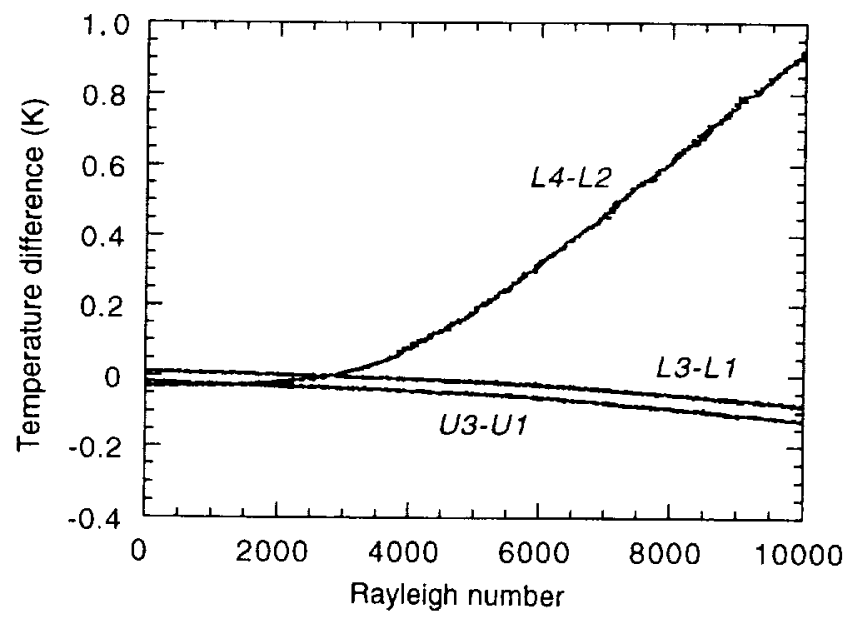

Fig. 3. Temperature differences of thermistors situated directly across from each other in the test cell versus Rayleigh number

rates. For $B_{0}=0$, hysteresis was observed in the measurement of $R a^{\prime}$ for heating and cooling rates $|\mathrm{d} T / \mathrm{d} t|>2 \times 10^{-3} \mathrm{~K} / \mathrm{s}$. That is, for heating rates larger than approximately $2 \times 10^{-3} \mathrm{~K} / \mathrm{s}$, the onset of convection occurred at Rayleigh numbers above $R a^{c}$, and for large cooling rates the transition to quiescence occurred below $R a^{c}$. For $B_{0}=0$, Experimental measurements of $R a^{i}$ found it to be independent of heating rates below $2 \times 10^{-3} \mathrm{~K} / \mathrm{s}$. For $B_{0} \neq 0$, hysteresis was observed in the measurement of $R a^{c}$, even for heating and cooling rates $|\mathrm{d} T / \mathrm{d} t|$ $<4 \times 10^{-4} \mathrm{~K} / \mathrm{s}$.

Figure 3 is a plot of the temperature differences of thermistors situated $180^{\prime \prime}$ across from each other versus Rayleigh number. For this particular experiment, the 8 signals recorded were from thermistors $L 1: L 4, U 1, U 3$, and thermocouples $T_{a}$ and $T_{b}$. The break in the slope of $L 4 L 2$ indicates the onset of heat transport by convection and the occurrence of $R a^{c}$. It has been predicted (Buell and Catton 1983) that for a vertical cylinder with an aspect ratio of 1 the flow mode which will occur immediately above $R a^{c}$ is a single non-axisymmetric meridional roll (a roll in the $r-z$ plane). The data in Fig. 3 are consistent with this prediction. The data indicate that a single roll exists predominantly in the $L 2, L 4 z$ plane. The $U 3-U 1$ and $L 3-L 1$ temperature differences remain nearly zero because the lines drawn between these thermistors are perpendicular to the direction of flow.

The measured value of $R a^{x}$ without a rotating magnetic field was 3800 . As shown in Table 2, this compares favorably to recent numerical simulations (Neumann 1990) and theoretical 
Table 2.

\begin{tabular}{lll}
\hline$R a^{i}$ & Method & Reference \\
\hline 3800 & Experimental & This work \\
3610 & Numerical & Neumann (1990) \\
3800 & Theoretical & Buell and Catton (1983) \\
6100 & Experimental & Weber (1988) \\
6000 & Experimental & Neumann (1986) \\
\hline
\end{tabular}

results (Buell and Catton 1983). The numerical and theoretical results shown in Table 2 assume a vertical cylinder with an aspect ratio of 1 and adiabatic sidewalls. Catton (1972) demonstrated that the thermal conductivity of the swidewalls can have a significant effect on $R a^{c}$. He defined a wall admittance $C=h k_{f} / d_{w} k_{w}$, where $k_{r}$ and $k_{w}$ are the thermal conductivities of the fluid and the wall and $d_{w}$ is the wall thickness. The wall admittaance of the present experiment $(C \approx 350)$ is such that the experimental data can be compared to theoretical calculations in the limit of adiabatic sidewalls.

Figure 4 is a plot of the derivative of the thermistor signals with respect to time versus Rayleigh number. The thermistors signals have been offset for clarity. By taking the derivative of the thermistor signals the transition to time-dependent convection becomes quite pronounced. A hysteresis effect was observed in the measurement of $R a^{t}$, even down to the lowest heating and cooling rates used, $|\mathrm{d} T / \mathrm{d} t|=1.2 \times 10^{-4} \mathrm{~K} / \mathrm{s}$. At the lowest cooling rate used, $R a^{t}$ occurred at an approximately $0.5 \mathrm{~K}$ lower temperature upon cooling than it did upon heating. Such hysteresis effects have been experimentally observed previously (see, for example, Krishnamurti 1973), and are most likely the result of finite amplitude instabilities. In the present experiment it was found that $R a^{\prime} \approx 7.3 R a^{\prime}$. This is comparable to the experimental results of Müller et al. (1984), who found $R a^{t} \approx 8 R a^{i}$. However, it is unclear why the value of $R a^{t}$ upon heating, $2.7 \times 10^{4}$, is significantly higher than the recent numerical result of $1.5 \times 10^{4}$ (Neumann 1990).

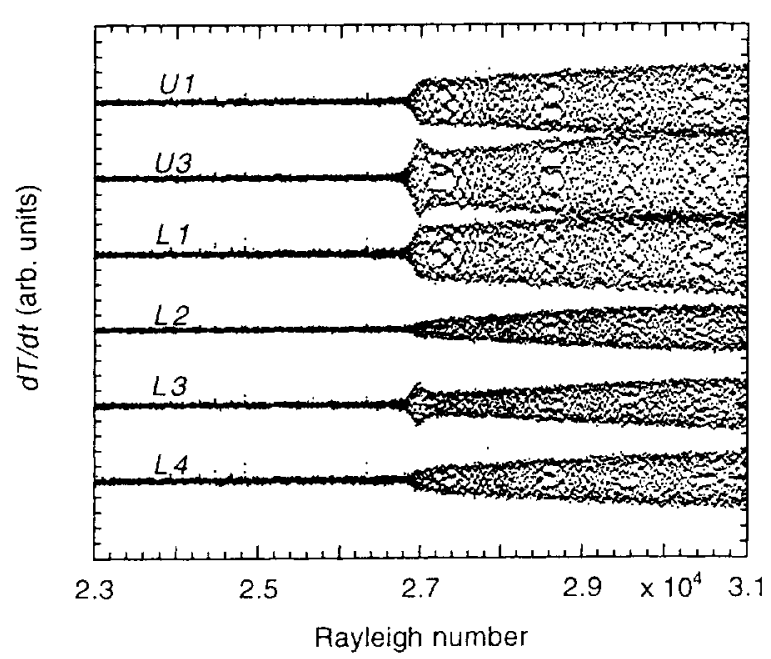

Fig. 4. Derivatives of thermistor temperatures with respect to time versus Rayleigh number. The transition to time-dependent flow is observed at $R a=2.7 \times 10^{4}$
4

\section{Flow transitions in a rotating magnetic field}

Consider a cylinder of liquid metal such as depicted in Fig. 2 under the influence of a magnetic field described by Eq. (1). The rotating magnetic field induces electric currents in the liquid which in turn interact with the field to produce an azimuthal electromagnetic force in the melt. This force drives an azimuthal fluid rotation in the cylinder. The strength of the magnetic field used in these experiments was such that the angular frequency of induced fluid rotation was always several orders of magnitude less than $60 \mathrm{~Hz}$, the frequency of the applied magnetic field. The degree of penetration of the field into the liquid is determined by the skin depth $\delta$ where

$\delta=\sqrt{\frac{2}{\mu \sigma(!)}}$

where $\mu$ is the magnetic permeability and $\sigma$ is the electrical conductivity. Here, the skin depth is larger than the diameter of the test cell and so the transverse magnetic field can be satisfactorily approximated as uniform and independent of $r$.

Two non-dimensional numbers are required to describe the fluid flow states that exist under the combination of buoyancydriven convection and convection driven by a rotating magnetic field. These non-dimensional numbers can be chosen as the Rayleigh number, defined in Eq. (2), and the Hartmann number, defined as

$H a=h B_{0} \sqrt{\frac{\sigma}{\rho v}}$

Experimentally, these numbers were varied by changing the temperature difference $T_{b}-T_{a}$ and the magnetic field strength $B_{0}$, respectively. As in the case when $B_{0}=0, R a^{c}$ and $R a^{t}$ are used to define the critical numbers at which the fluid flow transitions from one state to another. Howewver, an azimuthal component of fluid flow always exists in the presence of a rotating magnetic field. Therefore, $R a^{c}$ is now defined as being that value at which buoyancy-driven meridional flow exists and contributes significantly to convective heat transfer in the cylinder. $R a^{t}$ is now defined as that value at which time-dependent flow is observed. However, this time-dependent flow does not include that which is rotating around the cylinder with a constant azimuthal velocity. This time-dependent convection can be buoyancy-driven, driven by the rotating magnetic field, or driven by some combination of the two forces. Indeed, as will be shown, there is a critical value of $H a$ for which $R a^{t}=0$.

Four distinct flow regimes have been identified. These four regimes are defined solely by the presence or absence of buoyancy-driven convection and time-dependent convection. No attempt was made to subdivide the time-dependent flow regime (both oscillatory and turbulent) and quantify the various flow modes that can exist there.

\section{1}

\section{$\mathbf{R a}<\mathbf{R a}^{\prime}, \mathbf{R a}^{\prime} ; \mathrm{Ha}<3.8$}

The flow in this regime can be described as being in the laminar, time-independent convective state. The rotating magnetic field drives an azimuthal flow component with the flow velocity depending on the strength of the magnetic field. 
Experimentally, all of the thermistors in the liquid exhibit a smooth, time-independent reading. Although the thermistors do not register any temperature changes, a secondary meridional flow may exist. Indeed, as has been discussed previously (Davidson and Hunt 1987), a variation in the azimuthal force as a function of $z$, which may occur in a cylinder of finite height, can cause such a secondary flow. Also, because $R a<$ $R a^{c}$, the vertical temperature gradient is not strong enough for any buoyancy-driven flow to exist.

\section{2}

$\mathbf{R a}^{c}<\mathbf{R a}<\mathbf{R a}^{t} ; \mathbf{H a}<\mathbf{1}$

In this flow regime, the rotating magnetic field is relatively weak but still drives a laminar, azimuthal flow component. $R a$ is larger than $R a^{c}$, and so a buoyancy-driven meridional flow also exists. Figure 5 shows the transition between the regime described in Sect. 4.1 and the present one. The experimental conditions used to generate the transition shown in Fig. 5 are essentially the same as those used to generate the transition shown in Fig. 3. The major difference is that the data of Fig. 5 were obtained in the presence of a rotating magnetic field with $B_{0}=0.72 \mathrm{mT}$. Just as in the case when $H a=0$, above $R a^{c}$ a single, non-axisymmetric roll sets in. However, this single, non-axisymmetric meridional roll rotates around the cylinder, driven by the rotating magnetic field. The $L 3-L 1$ and $L 4-L 2$ thermistor signals have a near-sinusoidal response as a function of time. The $L 3-L 1$ signal is shifted $90^{\circ}$ with respect to the $L 4-L 2$ signal, and the maximum temperature measured at $L 4$ occurs before the maximum temperature measured at $L 3$. The angular velocity and rotational period can easily be calculated from data such as is shown in Fig. 5. The angular frequency of rotation of the single, non-axisymmetric roll increases with increasing magnetic field strength. It should also be noted that the critical Rayleigh number at which the transition observed in Fig. 5 occurs depends on the magnetic field strength, with $R a^{c}$ increasing as $\mathrm{Ha}$ increases.

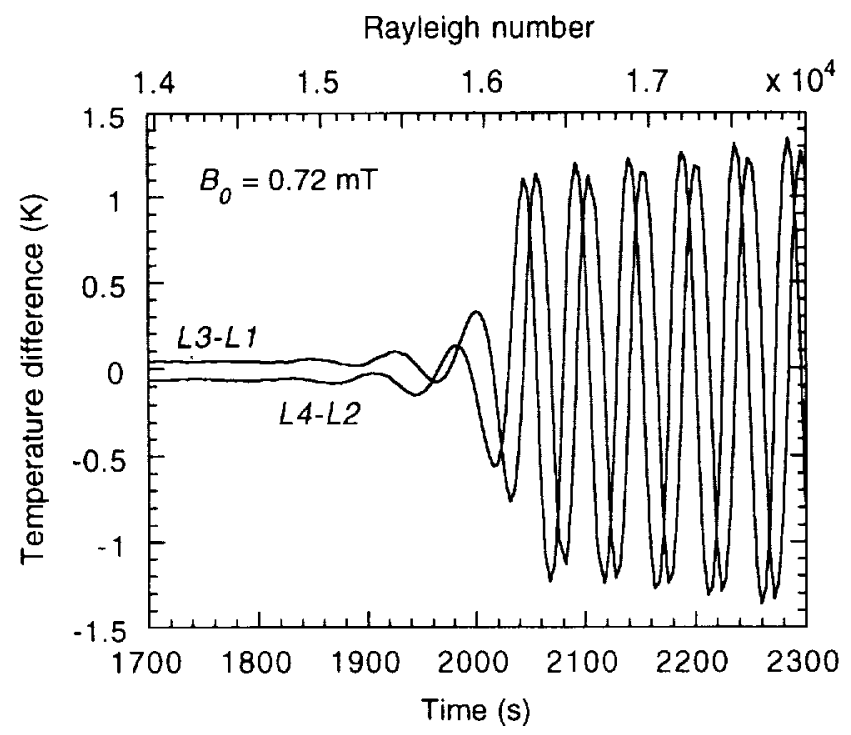

Fig. 5. Temperature differences of thermistors situated directly across from each other in the test cell versus time. The corresponding Rayleigh number is shown at the top of the figure. The rotating magnetic field strength is $0.72 \mathrm{mT}$

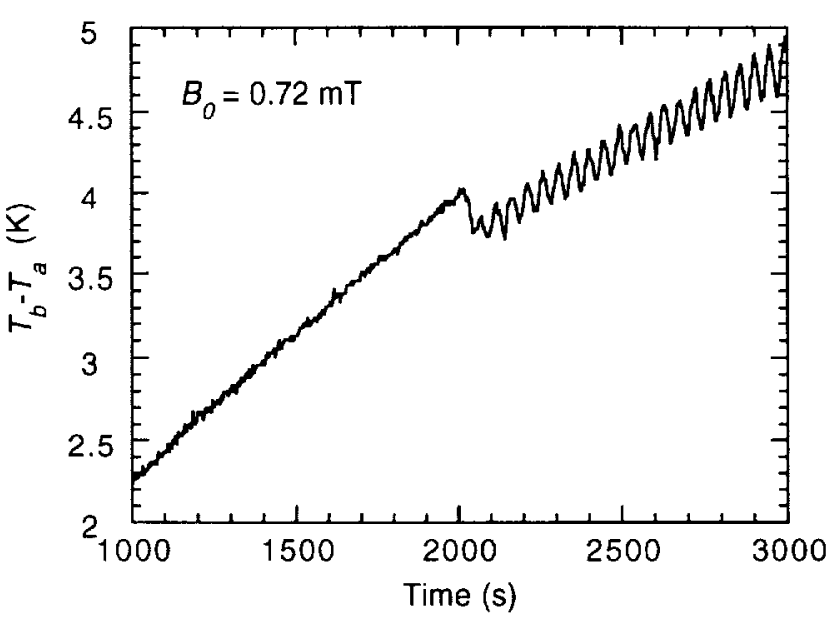

Fig. 6. Temperature differences between the bottom and top of the cell versus time. The decrease in $T_{b}-T_{a}$ at approximately $2000 \mathrm{~s}$ indicates the occurrence of the flow transition at $R a^{t}$

The data in Fig. 6 are from the same experimental run as those shown in Fig. 5. The difference in temperature between the top and bottom of the cell is plotted as a function of time. It can be clearly seen when the transition at $R a^{c}$ occurs. At approximately 2000 seconds, $T_{b}-T_{a}$ decreases slightly. It is at this point that buoyancy-driven meridional convection sets in and causes convective heat transport between the bottom and top of the test cell. After the transition, $\triangle T$ exhibits a sinusoidal characteristic. The frequency of this signal is the same as that observed for the thermistor temperature differences shown in Fig. 5. The thermocouples embedded in the copper plates at the top and bottom of the cell are off-center. Therefore, the observance of the sinusoidal signal indicates that the thermal conductivity of the copper plates is finite and that there is a small, time-dependent, radial temperature gradient across them.

The angular velocity of rotation was measured at several different magnetic fields, while keeping $R a$ constant. These data are plotted in Fig. 7. Even at the lowest magnetic fields

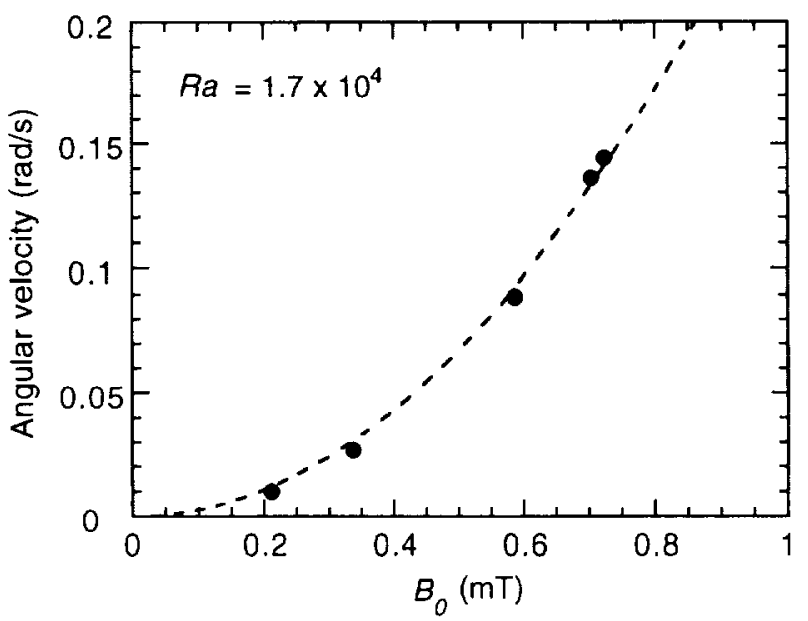

Fig. 7. Angular velocity of the meridional roll versus rotating magnetic field strength for $R a=1.7 \times 10^{4}$. The solid circles are the experimental data. The dashed line is a fit to the data assuming that the angular velocity of the roll is proportional to $B_{0}^{2}$ 
used, magnetically-driven azimuthal flow was observed. However, at sufficiently high magnetic fields, $R a^{c}$ becomes larger than $R a$, and the buoyancy-driven meridional flow ceases. At this point, the flow transitions to the regime described in Sect. 4.1. Thus, for $R a=1.7 \times 10^{4}$, the experimental data shown in Fig. 7 essentially cover the entire magnetic field range of this flow regime. The dashed line in Fig. 7 is a fit to the data assuming that the angular velocity is proportional to $B_{0}^{2}$. As described by Davidson and Hunt (1987), such a dependence is consistent with laminar flow. It should be noted, however, that what is plotted in Fig. 7 is not the angular flow velocity, but rather the angular velocity of the azimuthal rotation of the meridional roll. Indeed, no $r$ dependence was observed in the azimuthal rotation of the meridional roll, as would certainly be the case for the angular flow velocity.

\section{3}

\section{$\mathbf{R a}>\mathbf{R a}^{c}, \mathbf{R a}^{i}$; all $\mathbf{H a}$}

In this regime, the flow is in the time-dependent state. This flow can consist of two or more oscillatory modes, or, with increasing $R a$, turbulent flow. Because $R a>R a^{c}, H a$ is by definition not large enough to suppress buoyancy-driven meridional convection. This flow regime can be reached from that described in Sect. 4.2 by slowly increasing $R a$ until $R a>$ $R a^{t}$. Above the transition, the near-sinusoidal thermistor signals, such as are shown in Fig. 3, gain an additional buoyancy-driven time-dependent component. The occurrence of the transition can be clearly observed by taking the derivative of the signals with respect to time, which results in data plots similar to those shown in Fig. 4.

\section{4}

$\mathbf{R a}^{i}<\mathbf{R a}<\mathbf{R a} ; \mathbf{H a}>\mathbf{1}$

In this flow regime, $R a^{t}$ is always larger than $R a^{t}$. The combination of buoyancy and magnetically induced forces is strong enough to induce time-dependent convection, but $\mathrm{Ha}$ is large enough to suppress buoyancy-driven meridional convection. The effect of increasing $R a^{c}$ as the rotating magnetic field increases is similar to the situation descdribed by Chandrasekhar (1961), where the $R a^{c}$ of fluid in a rotating cylinder increases according to the Taylor number. Here, the external rotating cylinder is replaced by a rotating magnetic field, but the effects are similar. As $\mathrm{Ha}$ increases, $\mathrm{Ra}^{c}$ increases. In general, the time-dependence of the thermistor signals is driven by both electromagnetic and buoyancy forces. However, timedependent thermistor signals were always observed for $\mathrm{Ha}>3.8$, even with $R a=0$. At $R a^{\prime}$, which depends on both $H a$ and $R a$, the magnetic field drives the fluid to a high enough velocity that it transitions from the laminar to time-dependent state.

\section{5}

\section{Summary}

A cylindrical test cell was constructed to study liquid metal flows in a rotating magnetic field. The fluid flow was inferred from temperature measurements of thermistors immersed in liquid gallium. The measured value of $R a^{c}$ at zero magnetic field was in good agreement with recent theoretical and numerical results. Immediately above $R a^{i}$, the thermistor signals indicated a signal non-axisymmetric roll. Such a roll was consistent with previous theoretical predictions (Buell and
Catton 1983) for a cylinder with an aspect ratio of 1 , and was observed experimentally by Müller et al. (1984). With the magnetic field on, four distinct flow regimes were identified. In two of these regimes, laminar flow was observed. These are the flow regimes in which single crystal growth might be expected to be most useful. The results indicated that a rotating magnetic field can increase $R a^{c}$ and extend the range over which laminar flow is obtained. However, it should be noted that laminar flow could only be obtained for Hartmann numbers less than approximately 3.8. Above that value the flow was always observed to be time-dependent. A theoretical description of the critical Rayleigh number's dependence on rotating magnetic field strength is the subject of future work.

\section{References}

Barmin IV; Egorov AV; Filatov IG; Senchenkov AS; Benz KW; Lexow B; Salk M; Hofmạnn P; Sickinger P; Gelfgat JM; Sorkin MZ; Matioukhin DG (1992) CdTe crystal growth by THM with a rotating magnetic field. Proc. of the VIIIth European Symposium on Materials and Fluid Sciences in Microgravity, Brussels, Belgium, 315320

Brückner FU; Schwerdtfeger K (1994) Single crystal growth with the Czochralski method involving rotational electromagnetic stirring of the melt. J Cryst Growth 139:351 356

Buell JC; Catton I (1983) The effect of wall conduction on the stability of a fluid in a right circular cylinder heated from below. J Heat Transfer ASME 105: 255260

Catton I (1972) Effect of wall conduction on the stability of a fluid in a rectangular region heated from below. J Heat Transfer ASME 94: $446 \quad 452$

Chandrasekhar RS (1961) Hydromagnetic and Hydrodynamic Stability. Oxford: Clarendon Press

Cusack N; Kendall P (1960) A note on the viscosity and resistivity of liquid gallium. Proc Phys Soc 75: 309311

Davidson P; Hunt JCR (1987) Swirling recirculating flow in a liquid metal column generated by a rotating magnetic field. J Fluid Mech 185: $67 \cdot 106$

Dold P; Cröll A; Kaiser Th; Salk M; Benz KW (1995) Semiconductor crystals grown under microgravity and in magnetic fields. AlAA paper 95-0264, AIAA 33rd Aerospace Sciences Meeting, Reno, Nevada

Doronin VI; Dremov VV; Kapusta AB (1973) Measurement of the characteristics of the magnetohydrodynamic flow of mercury in a closed cylindrical vessel. Magntinaya Gidrodinamika 3: 138-140

Gelfgat YuM; Gorbunov LA; Kolevzon V (1993) Liquid metal flow in a finite-length cylinder with a rotating magnetic field. Exp Fluids 15 : $411-416$

Grosse AV (1961) The viscosity of liquid metals and an empirical relationship between their activation energy of viscosity and their melting point. J Inorg N Chem 23: $333 \cdot 339$

Hoather WH (1936) The density and coefficient of expansion of liquid gallium over a wide range of temperature. Proc Phys Soc 48: 699707

Iida T; Guthrie RIL (1988) The physical properties of liquid metals. Oxford: Clarendon Press

Krishnamurti R (1973) Some further studies on the transition to turbulent convection. J Fluid Mech 60: 285 303

Müller G; Neumann G (1983) Investigation of convective flows in model systems of directional solidification configurations. Proc. of the IVth European Symposium on Materials Sciences under Microgravity, Madrid, Span, 285294

Müller G; Neumann G; Weber W (1984) Natural convection in vertical bridgman configurations. J Cryst Growth 70: $78 \cdot 93$

Nalgiev AV; Ibragimov KI (1974) Surface tension and density in the tin-gallium system. Russian J Phys Chem 48: 754756

Neumann G (1986) Dissertation, Universität Erlangen-Nürnberg

Neumann G (1990) Three-dimensional numerical simulation of buoyancy-driven convection in vertical cylinders heated from below. J Fluid Mech 214: 559578

Powell RL; Childs GE (1972) American Institute of Physics Handbook, p. 4 153, New York: McGraw-Hill

Weber W (1988) Dissertation, Universität Erlangen-Nürnberg 
